\title{
A ROTATING DISK MODEL FOR A QUASAR
}

\author{
F.D. KAHN \\ (Astronomy Dept., University of Manchester)
}

It has often been suggested that the energy supply of a quasar is derived from a central body with a mass of $10^{9}-10^{10} M_{\odot}$. However, it is known that such bodies have no stable and static equilibrium configurations beyond a relatively low degree of condensation. Only a very limited amount of energy can be extracted before instability sets in. Basically the instability is due to the dominance of radiation pressure in supporting the mass against its self-gravitation.

But if a massive object is in rapid rotation, then it is partly supported by centrifugal force, and is much less liable to an instability of this kind. It is therefore interesting to investigate the properties of a rotating disk-like object with a mass of the order of $10^{9}-10^{10} M_{\odot}$, and to see whether its overall properties match those of any quasar. The most important parameters are $2 \sigma_{0}$, the mass per unit area of the disk, and $Z$, its half-thickness. In the case of the quasar $3 C 273 \mathrm{~B}$, assumed to be at a distance of $450 \mathrm{Mpc}$, the most suitable pair of values are $\sigma_{0}=10^{7} \mathrm{gm} / \mathrm{cm}^{2}$ and $Z=10^{16} \mathrm{~cm}$.

With these parameters the following properties are predicted for the model. The energy output is derived from the collapse of the disk towards its central plane, but this is slow and proceeds through a sequence of quasi-static states. The outflow of radiation is checked by electron opacity, and radiation pressure is much more important than gas pressure. The matter in the disk is hot enough to be fully ionized but the temperature in its central layer is only about $3 \times 10^{5} \mathrm{deg} \mathrm{K}$. No important nuclear reactions can therefore take place, and so the object does not change its composition as it evolves.

The radiative loss per unit mass must equal $4 \pi G c / \kappa=6 \times 10^{4} \mathrm{erg} \mathrm{g}^{-1} \mathrm{~s}^{-1}$ everywhere, where $\kappa=$ electron opacity. This necessarily follows when radiation pressure and electron opacity dominate. Thus the outer layers, which are cooler, must release radiation at the same rate as the inner layers, which are much hotter. The outer layers thus overcool, and convection begins.

Now in a structure that is supported by radiation pressure, the temperature everywhere is determined simply in terms of the pressure, and is uniform at any level. The rising and the falling convective streams are therefore equally hot. Because of this there is no heat exchange between them, and the convected bubbles may have a much longer lifetime than they do when gas pressure dominates. Perhaps the fluctuations in brightness of a quasar are due to the irregular arrival of large bubbles at its surface.

Perek (ed.), Highlights of Astronomy, 389-390. () I.A.U. 
Finally, the predicted lifetime for the object is $3 \times 10^{5}$ years, the colour temperature in the visible is about $50000 \mathrm{deg} \mathrm{K}$, and the natural period of the disk for dynamical vibrations about 10 years.

\section{DISCUSSION}

E.M. Burbidge: Is not most of your energy thermal? How do you explain the observed optical polarization?

$K a h n$ : Something will obviously have to be added to the theory.

G. Burbidge: Most of the observed energy is in polarized infrared.

Bidelman: Have you considered element production?

Kahn: No. 